\title{
Bevacizumab plus chemotherapy as salvage treatment in chemorefractory patients with metastatic colorectal cancer
}

This article was published in the following Dove Press journal:

OncoTargets and Therapy

24 January 2013

Number of times this article has been viewed

\author{
Ravit Geva ${ }^{1,2}$ \\ Loredana Vecchione ${ }^{2}$ \\ Sabine Tejpar ${ }^{2}$ \\ Hubert Piessevaux ${ }^{3}$ \\ Eric Van Cutsem ${ }^{2}$ \\ Hans Prenen ${ }^{2}$ \\ 'Gastrointestinal Malignancies Service, \\ Oncology Division, Tel Aviv Sourasky \\ Medical Center, Tel Aviv, Israel; \\ ${ }^{2}$ Department of Gastroenterology, \\ Digestive Oncology Unit, University \\ Hospitals Leuven, Leuven, Belgium; \\ ${ }^{3}$ Department of Gastroenterology, \\ Cliniques Universitaires Saint-Luc, \\ Brussels, Belgium
}

Purpose: The combination of chemotherapy and bevacizumab, a monoclonal antibody targeting the vascular endothelial growth factor, is consistently being used as first- and secondline treatment in patients with metastatic colorectal cancer (mCRC). There is little data of the activity of bevacizumab in chemorefractory mCRC patients. The aim of this retrospective single center study was to evaluate the activity of bevacizumab combined with chemotherapy in this study population.

Methods: Forty-six consecutive mCRC patients treated in the University Hospital Gasthuisberg (Leuven, Belgium) receiving bevacizumab in advanced lines following failure of conventional chemotherapy were included in this study. Treatment regimen consisted of bevacizumab $5 \mathrm{mg} / \mathrm{kg}$ in combination with leucovorin, 5-fluorouracil, and oxaliplatin (FOLFOX) or leucovorin, 5-fluorouracil, and irinotecan (FOLFIRI).

Results: Bevacizumab plus chemotherapy was used in third-line treatment in eight $(17 \%)$ patients and in fourth-line treatment or more in 38 patients $(83 \%)$. All patients previously failed irinotecan-based chemotherapy, 44 (96\%) failed oxaliplatin-based regimens, and $40(87 \%)$ failed treatment with cetuximab. Bevacizumab was given in combination with irinotecan-based chemotherapy in 36 patients, oxaliplatin-based chemotherapy in nine patients, and with single agent 5-fluorouracil in one patient. Objective response was demonstrated in ten patients $(22 \%)$ and disease control in $38(83 \%)$ with a median progression-free survival of 8.9 months and a median overall survival of 13.8 months. Only four patients experienced grade III and above bevacizumab-related toxicity.

Conclusion: Taking into account the retrospective nature of the study which can influence the selection of patients, bevacizumab given in advanced lines after failure of conventional chemotherapy and antiepidermal growth factor receptor agents can result in high disease control rates in patients with $\mathrm{mCRC}$.

Keywords: colorectal cancer, bevacizumab, chemorefractory

\section{Introduction}

Although the incidence and the mortality of colorectal cancer (CRC) have decreased over the years, ${ }^{1}$ it still remains the third most frequently diagnosed cancer and the second leading cause of cancer death worldwide. ${ }^{2}$ About $40 \%-50 \%$ of newly diagnosed patients have metastatic disease and, despite therapeutic advances, the prognosis for metastatic $\mathrm{CRC}(\mathrm{mCRC})$ patients still remains poor. The majority of patients with $\mathrm{mCRC}$ cannot be cured, although a subset with isolated liver and/or lung metastases are potentially curable by surgery. The introduction into clinical practice of cytotoxic agents such as oxaliplatin and irinotecan has improved the response rates, progression-free survival (PFS), and
University Hospitals Leuven, Department of Gastroenterology, Digestive Oncology Unit, Herestraat 49, B3000 Leuven,

Belgium

Tel +3216344218

Fax +3216344419

Email hans.prenen@uzleuven.be 
overall survival (OS) compared with single agent 5-fluorouracil (5-FU). ${ }^{3-6}$ Moreover, the combination of chemotherapy with targeted biological agents such as antiepidermal growth factor receptor (anti-EGFR) and antivascular endothelial growth factor (anti-VEGF) monoclonal antibodies has increased the median OS to nearly 24 months. $^{7-11}$

In mCRC, bevacizumab has demonstrated activity both in a first- and second-line setting in combination with either irinotecan- or oxaliplatin-based schedules. ${ }^{7,8,12,13}$ An important clinical issue is whether bevacizumab should be continued after failing a first-line bevacizumab-containing regimen. This question was studied in the recently presented ML18147 (TML) trial, which revealed a significant improvement in PFS and OS. ${ }^{14}$ Two other agents targeting VEGF - regorafenib and aflibercept - have also recently shown promise for the treatment of mCRC. ${ }^{15,16}$

Despite the demonstrated activity and efficacy of bevacizumab, its role in chemorefractory mCRC patients "to reverse chemoresistance" - is still controversial.

Therefore, the aim of this retrospective analysis was to investigate the benefit of bevacizumab as a third-line and further treatment in chemorefractory mCRC patients after documented failure of 5-FU, oxaliplatin, irinotecan, and anti-EGFR agents.

\section{Material and methods Patient characteristics}

Patients with histologically proven metastatic adenocarcinoma of the colon or rectum that received bevacizumab after progression to 5-FU, oxaliplatin, and irinotecan were eligible for the current retrospective analysis. Patients who progressed after anti-EGFR or who experienced unacceptable toxicity from one of these chemotherapeutic agents were also included. Evaluable metastatic lesions at treatment initiation and at least one evaluation assessment during the antiangiogenetic treatment were required.

Other common eligible criteria were: an Eastern Collaborative Oncology Group performance status of less than two, adequate hematological function (hemoglobin $>9 \mathrm{~g} / \mathrm{dL}$, neutrophil count $>1500 / \mathrm{mm}^{3}$, platelet count $>100,000 / \mathrm{mm}^{3}$ ), adequate hepatic function (total bilirubin less than 1.5 times upper limit of normal range, aspartate aminotransferase and alanine aminotransferase less than five times the upper limit of normal), and renal function with serum creatinine less than five times the upper limit of normal. History of major surgery within 28 days, thrombotic or bleeding events within 6 months, clinically significant cardiovascular disease including hypertension and therapeutic anticoagulation, and presence of brain metastases were considered as exclusion criteria. Patients treated with bevacizumab between the years 2004 and 2011 were selected and prospectively registered in a computerized database. When the first cycle of bevacizumab was administered, the following data were collected and analyzed: age and performance status (according to Eastern Collaborative Oncology Group criteria), gender, primary tumor site (colon or rectum), previous anticancer drugs received, and corresponding tumor response.

\section{Treatment regimen}

Patients were treated with bevacizumab at a dose of $5 \mathrm{mg} / \mathrm{kg}$ every 2 weeks following the common protocol of 90 minutes for the first infusion, 60 minutes for the second, and 30 minutes for the following administrations. Every 2 weeks, after bevacizumab infusion, patients received either leucovorin, 5-FU, and oxaliplatin (FOLFOX; 2-hour infusion of $85 \mathrm{mg} / \mathrm{m}^{2}$ of oxaliplatin, followed by a bolus of $400 \mathrm{mg} / \mathrm{m}^{2} 5-\mathrm{FU}, 2$-hour infusion of $400 \mathrm{mg} / \mathrm{m}^{2}$ leucovorin and a 46-hour infusion of $2400 \mathrm{mg} / \mathrm{m}^{2} 5-\mathrm{FU}$ ) or leucovorin, 5-FU, and irinotecan (FOLFIRI; 90 minutes of $180 \mathrm{mg} / \mathrm{m}^{2}$ irinotecan, followed by a bolus of $400 \mathrm{mg} / \mathrm{m}^{2}$ 5-FU, 2-hour infusion of $400 \mathrm{mg} / \mathrm{m}^{2}$ leucovorin, and a 46-hour infusion of $2400 \mathrm{mg} / \mathrm{m}^{2} 5 \mathrm{FU}$ ). Dose reductions were applied in cases of grade III/IV toxicities, according to World Health Organization toxicity criteria.

\section{Tumor evaluation}

To be considered evaluable, patients must have received at least four administrations of bevacizumab in combination with chemotherapy. Patients were evaluated by using contrast-enhanced spiral computed tomography at baseline and every 2-3 months until progression. Confirmatory computed tomography scans for response or progressive disease are not done in daily practice and were therefore not available. Moreover, the evaluations were first analyzed by the radiologist and then by the attending physician upon treatment decision. In cases of conflicting assessments, the computed tomography scans were reevaluated by the authors with the application of Response Evaluation Criteria In Solid Tumors (RECIST) 1.1 criteria.

\section{Statistical analysis}

Descriptive statistics was used to characterize the patients. Kaplan-Meier curves were used to plot OS and PFS.

\section{Results}

A total of 46 chemorefractory patients who received bevacizumab in combination with either FOLFOX or 
FOLFIRI between 2004 and 2011 were retrospectively analyzed. The main characteristics of the patient population are reported in Table 1.

Eight $(17.4 \%)$ patients received bevacizumab in third-line treatment while $38(82.6 \%)$ patients received the antiangiogenic drug in fourth-line or in further lines of treatment. All the patients had a good Eastern Collaborative Oncology Group performance status (less than two) at the beginning of the bevacizumab treatment. Twenty (43.5\%) patients had a rectum primary location, while $26(56.5 \%)$ patients had a colon primary location. All patients had previously failed an irinotecan-based chemotherapy regimen. Forty-four $(95.7 \%)$ patients failed an oxaliplatin-based regimen and 40 (87\%) failed a cetuximab-based regimen. Thirty-six patients $(78.3 \%)$ received bevacizumab in combination with irinotecan-based chemotherapy, while nine patients received bevacizumab in combination with FOLFOX. One patient received the antiangiogenic treatment in combination with single agent 5-FU.

Objective response rate was observed in ten patients $(21.7 \%)$, while disease control rate was observed in 38 patients $(82.6 \%)$.

Median time of treatment since diagnosis of metastatic disease was 34.7 months.

With a median follow-up of 10.6 months (range 3-33.7 months) from the beginning of bevacizumab, the median PFS was 8.9 months (95\% confidence interval 5.5-12.3) (Figure 1) and the median OS was 13.8 months (95\% confidence interval 11.2-16.4) (Figure 2). No statistically

Table I Patient demographics

\begin{tabular}{ll}
\hline & Number (\%) \\
\hline Gender & \\
Male & $30(65)$ \\
Female & $16(35)$ \\
Location & \\
Rectum & $20(43)$ \\
Colon & $26(57)$ \\
Previous metastatic treatment & \\
Oxaliplatin & $44(96)$ \\
Irinotecan & $46(100)$ \\
Anti-EGFR & $40(87)$ \\
Chemotherapy backbone with bevacizumab & \\
Oxaliplatin & $9(20)$ \\
Irinotecan & $36(78)$ \\
Single 5-FU & $1(2)$ \\
Line of bevacizumab & \\
3 & $8(17)$ \\
4 & $27(59)$ \\
$5-7$ & 1 I (24) \\
\hline
\end{tabular}

Abbreviations: 5-FU, 5-flurouracil; EGFR, epidermal growth factor receptor.

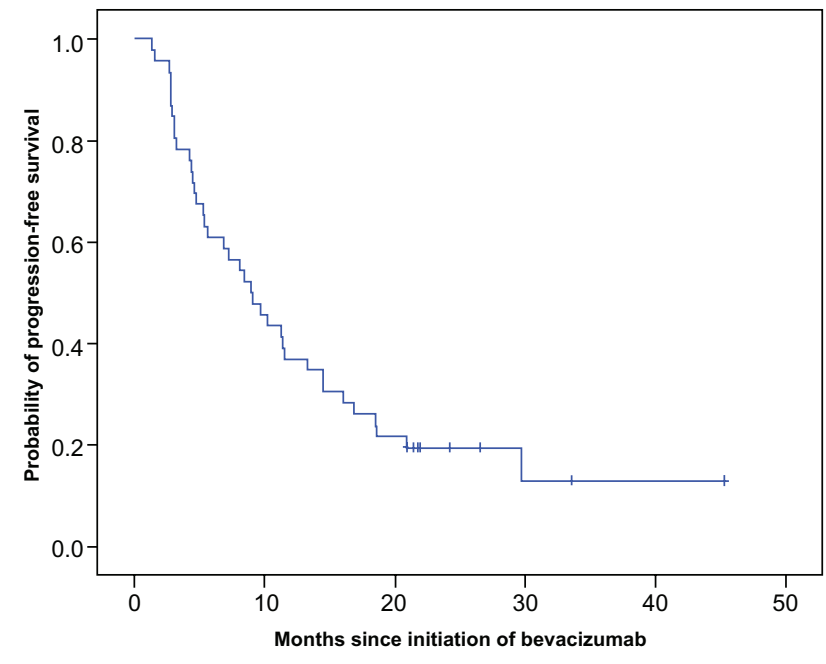

Figure I Progression-free survival on bevacizumab with chemotherapy. Note: Median progression-free survival 8.9 months, 95\% confidence interval 5.512.3 months.

significant differences in terms of PFS and OS for clinical variables such as gender, primary tumor location, and line of bevacizumab administration were observed.

Four $(8.7 \%)$ out of 46 patients experienced grade III and above bevacizumab-related toxicities; in two of them, hemoptysis resulted in death.

\section{Discussion}

Bevacizumab is a recombinant humanized monoclonal antibody that binds all isoforms of VEGF-A, ${ }^{17}$ thus preventing its interaction with receptors on endothelial cells and angioblasts. Angiogenesis, mainly regulated by VEGF receptor-2, is required for tumor growth and metastases. ${ }^{18}$

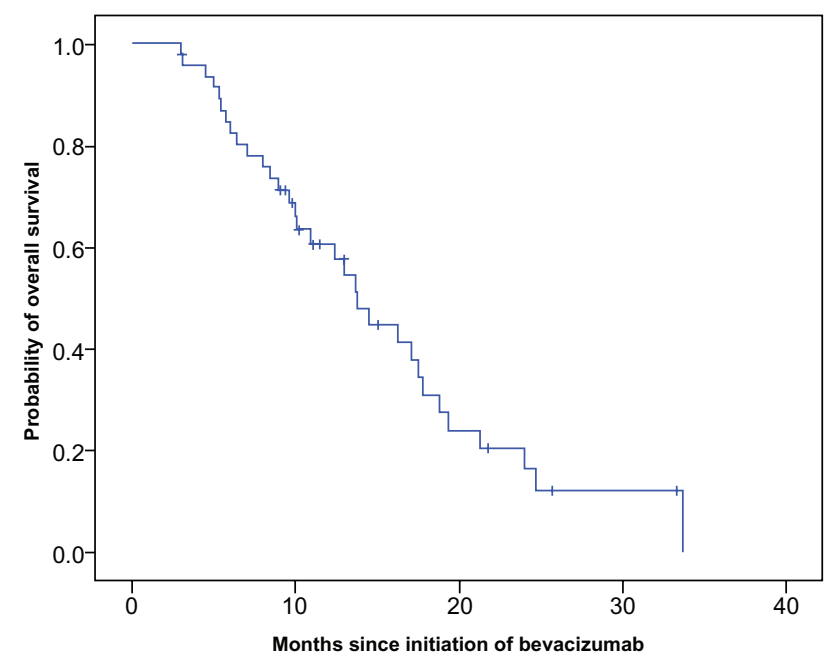

Figure 2 Overall survival on bevacizumab with chemotherapy.

Note: Median overall survival 13.8 months, 95\% confidence interval II.216.4 months. 
A wide range of human solid tumors have high VEGF expression, which correlates with worse prognosis and increased incidence of disease recurrence. ${ }^{19}$ By binding VEGF-A, bevacizumab is able to block the signal transduction cascade responsible for angiogenesis, thus preventing neovascularization and remodeling the existing vasculature through apoptosis of endothelial cells, leading to a decrease in vessel diameter, density, and permeability. ${ }^{20}$ Preclinical studies have demonstrated its capability to inhibit the growth of human tumor xenografts, ${ }^{21}$ while subsequent clinical trials have established its efficacy in solid tumors. ${ }^{22}$

In particular, when administered as first-line treatment for $\mathrm{mCRC}$ patients in combination with 5-FU and irinotecanbased chemotherapy, bevacizumab improves both PFS (10.6 versus 6.2 months) and OS (20.3 versus 15.6 months) compared to chemotherapy alone. ${ }^{8}$ Benefit in terms of OS and PFS has also been observed in combination with 5-FU alone ${ }^{23}$ and in combination with oxaliplatin-based regimens either as first-line treatment ${ }^{12,24}$ or second-line treatment. ${ }^{13}$ Moreover, its activity after failure of a previous bevacizumabcontaining regimen has recently been shown in the TML study, in which 820 patients with mCRC progressing beyond first-line chemotherapy with bevacizumab were randomly assigned to chemotherapy with or without bevacizumab. ${ }^{14}$ Continuation of bevacizumab was associated with a significant improvement in PFS (median 5.7 versus 4.1 months) and OS (median 11.2 versus 9.8 months). This data was confirmed in the Bevacizumab Beyond Progression (BEBYP) trial, a smaller Italian randomized Phase II trial that was terminated early after the TML results were available. ${ }^{25} \mathrm{~A}$ total of 184 patients were randomized to second-line chemotherapy with FOLFOX or FOLFIRI alone or in combination with bevacizumab. The study met its primary endpoint with a median PFS of 4.97 months in the chemotherapy alone arm and 6.77 months in the bevacizumab arm. OS data were not mature yet at the time this study was presented. Both trials validate the use of bevacizumab beyond progression.

In chemorefractory mCRC patients, OS is approximately 4 months. ${ }^{11}$ Several studies have investigated the question of a suitable chemotherapy regimen for these chemorefractory patients. Cetuximab has been shown to be effective in combination with irinotecan in irinotecan-refractory mCRC patients. ${ }^{26}$ The goal of the current retrospective analysis was then to investigate whether bevacizumab was also able to reverse chemoresistance in chemorefractory $\mathrm{mCRC}$ patients after documented failure of 5-FU, oxaliplatin, irinotecan, and anti-EGFR agents. To date, only a few studies have addressed this question, ${ }^{27-30}$ although there are no randomized clinical trials available regarding the role of bevacizumab-based chemotherapy in chemorefractory mCRC patients. The first study to address this question was a Phase II trial by Chen et al which included a total of 350 patients, refractory to both oxaliplatin- and irinotecan-based regimens. ${ }^{28}$ None of these patients were treated with anti-EGFR agents. This trial was initiated to provide access to bevacizumab when the agent was still investigational, but had already demonstrated a significant survival advantage. ${ }^{8}$ Median PFS was 3.5 months and OS was 9.0 months. A retrospective analysis by Kang et al also reported on the efficacy of bevacizumabcontaining third-line chemotherapy with either FOLFOX or FOLFIRI in patients previously progressing under FOLFOX and FOLFIRI and demonstrated a median PFS and OS of 5.3 and 9.5 months, respectively. ${ }^{30}$ Two other trials also investigated the value of bevacizumab in heavily pretreated mCRC patients. Vincenzi et al investigated the combination of bevacizumab with the continuous infusion of 5-FU (de Gramont regimen) in patients with oxaliplatin-, irinotecan-, and cetuximab-refractory mCRC patients. ${ }^{29} \mathrm{~A}$ total of 48 patients were included in this Phase II trial. Median time to disease progression was 3.5 months and median survival was 7.7 months. Moreover, Lievre et al reported a median PFS and OS of 9.7 months and 18.4 months, respectively, in 31 chemorefractory patients treated with a combination of bevacizumab with either FOLFIRI or FOLFOX. ${ }^{27}$ Sixtyone percent of these patients were also pretreated with cetuximab. The results of the current retrospective analysis are consistent with Lievre et al, although the percentage of patients pretreated with an anti-EGFR inhibitor in the current study was $87 \%$.

Together with previously published studies, the current data reveal that introducing bevacizumab in a very late phase of therapy in mCRC patients may lead to tumor control and improved survival. Whether bevacizumab needs to be combined with a doublet (FOLFOX/FOLFIRI) or with single agent 5-FU is not clear, but it seems that both options are acceptable. One of the main concerns in the current study is its retrospective nature and therefore its use of highly selected patients. Still, it seems that there is a subset of chemorefractory- and bevacizumab-naive patients that might have a clear benefit from adding bevacizumab as an advanced line of treatment. In patients previously treated with the same chemotherapy regimen, adding bevacizumab led to a disease stabilization rate of more than $80 \%$, which suggests that antiangiogenic therapy might circumvent 
chemotherapy resistance. Furthermore, it cannot be ruled out that pretreatment with an EGFR inhibitor might also lead to more sensitivity of antiangiogenic agents.

Preclinical data demonstrates that overexpression of VEGF renders tumor cells resistant to EGFR antibody-based therapy. ${ }^{31}$ Also, more recent work from Bianco et al revealed that VEGF receptor-1 was overexpressed in cells resistant to cetuximab. ${ }^{32}$ Experiments silencing VEGF receptor-1 in cetuximab-resistant cells restored sensitivity to cetuximab, whereas overexpression of VEGF receptor-1 in cetuximabsensitive cells conferred resistance to cetuximab. Collectively, these data suggest that altered angiogenesis could serve as a potential mechanism of resistance to anti-EGFR therapy and as a consequence pretreatment with anti-EGFR agent might render those tumors more sensitive to antiangiogenic agents. However, this hypothesis still needs to be validated in a prospective clinical trial.

Recently, regorafenib has emerged as another therapeutic option in chemorefractory mCRC patients. Regorafenib is an oral multikinase inhibitor which targets angiogenic, stromal, and oncogenic tyrosine kinases such as VEGF receptor-1/3, Tie-2, platelet-derived growth factor receptor- $\beta$, fibroblast growth factor receptor, Kit, Ret, and B-Raf. ${ }^{33}$ In the drug's pivotal Phase III randomized trial (Patients With mCRC Treated With Regorafenib or Placebo After Failure of Standard Therapy; CORRECT), median OS was 6.4 months in the regorafenib group and 5.0 months in the placebo group. ${ }^{15}$ On September 27, 2012 the Food and Drug Administration approved regorafenib as the first small-molecule kinase inhibitor to treat patients with mCRC. Whether the mechanism of action of regorafenib in colon cancer is an inhibition of angiogenesis or a direct blockage of a specific tyrosine kinase is currently unknown.

The authors would also like to stress that the exact mechanism by which bevacizumab enhances the efficacy of chemotherapy is still not well understood. It has been proposed that normalization of the tumor vasculature, reducing intratumoral pressure, and allowing better delivery of chemotherapy to the tumor might be responsible for maximizing antitumor activity. ${ }^{34}$

Finally, in light of the recent evolution of identifying molecular subtypes in colon cancer based on gene expression profile, mutation analysis, copy number variation, methylation, and micro ribonucleic acids, it will be important to identify the subgroup of patients that will benefit from treatment with an antiangiogenic agent, particularly because it has been clear from several randomized studies that the benefit of treating an unselected population with an antiangiogenic agent only leads to a small increase in PFS and OS. ${ }^{8,12}$ Based on the current retrospective analysis, it can be hypothesized that there are probably subgroups that might have more benefit when treated with an antiangiogenic agent.

\section{Conclusion}

This study confirms the activity of bevacizumab combined with chemotherapy in chemorefractory- and bevacizumabnaive $\mathrm{mCRC}$ patients who have failed prior chemotherapy with 5-FU, irinotecan, oxaliplatin, and anti-EGFR agents.

\section{Disclosure}

The authors report no conflicts of interest in this work.

\section{References}

1. Cheng L, Eng C, Nieman LZ, Kapadia AS, Du XL. Trends in colorectal cancer incidence by anatomic site and disease stage in the United States from 1976 to 2005. Am J Clin Oncol. 2011;34(6):573-580.

2. Siegel R, Ward E, Brawley O, Jemal A. Cancer statistics, 2011: the impact of eliminating socioeconomic and racial disparities on premature cancer deaths. CA Cancer J Clin. 2011;61(4):212-236.

3. de Gramont A, Figer A, Seymour M, et al. Leucovorin and fluorouracil with or without oxaliplatin as first-line treatment in advanced colorectal cancer. J Clin Oncol. 2000;18(16):2938-2947.

4. Douillard JY, Cunningham D, Roth AD, et al. Irinotecan combined with fluorouracil compared with fluorouracil alone as first-line treatment for metastatic colorectal cancer: a multicentre randomised trial. Lancet. 2000;355(9209):1041-1047.

5. Goldberg RM, Sargent DJ, Morton RF, et al. A randomized controlled trial of fluorouracil plus leucovorin, irinotecan, and oxaliplatin combinations in patients with previously untreated metastatic colorectal cancer. J Clin Oncol. 2004;22(1):23-30.

6. Saltz LB, Cox JV, Blanke C, et al; Irinotecan Study Group. Irinotecan plus fluorouracil and leucovorin for metastatic colorectal cancer. $N$ Engl J Med. 2000;343(13):905-914.

7. Van Cutsem E, Rivera F, Berry S, et al. Safety and efficacy of first-line bevacizumab with FOLFOX, XELOX, FOLFIRI and fluoropyrimidines in metastatic colorectal cancer: the BEAT study. Ann Oncol. 2009;20(11):1842-1847.

8. Hurwitz H, Fehrenbacher L, Novotny W, et al. Bevacizumab plus irinotecan, fluorouracil, and leucovorin for metastatic colorectal cancer. N Engl J Med. 2004;350(23):2335-2342.

9. Van Cutsem E, Kohne CH, Hitre E, et al. Cetuximab and chemotherapy as initial treatment for metastatic colorectal cancer. $N$ Engl J Med. 2009;360(14):1408-1417.

10. Van Cutsem E, Kohne CH, Lang I, et al. Cetuximab plus irinotecan, fluorouracil, and leucovorin as first-line treatment for metastatic colorectal cancer: updated analysis of overall survival according to tumor KRAS and BRAF mutation status. J Clin Oncol. 2011;29(15):2011-2019.

11. Amado RG, Wolf M, Peeters M, et al. Wild-type KRAS is required for panitumumab efficacy in patients with metastatic colorectal cancer. J Clin Oncol. 2008;26(10):1626-1634.

12. Saltz LB, Clarke S, Diaz-Rubio E, et al. Bevacizumab in combination with oxaliplatin-based chemotherapy as first-line therapy in metastatic colorectal cancer: a randomized phase III study. J Clin Oncol. 2008;26(12):2013-2019.

13. Giantonio BJ, Catalano PJ, Meropol NJ, et al. Bevacizumab in combination with oxaliplatin, fluorouracil, and leucovorin (FOLFOX4) for previously treated metastatic colorectal cancer: results from the Eastern Cooperative Oncology Group Study E3200. J Clin Oncol. 2007;25(12):1539-1544. 
14. Arnold D, Andre T, Bennouna J, et al. Bevacizumab (BEV) plus chemotherapy $(\mathrm{CT})$ continued beyond first progression in patients with metastatic colorectal cancer (mCRC) previously treated with BEV plus CT: results of a randomized phase III intergroup study (TML study) [abstract]. J Clin Oncol. 2012;30 Suppl:CRA3503.

15. Grothey A, Sobrero AF, Siena S, et al. Results of a phase III randomized, double-blind, placebo-controlled, multicenter trial (CORRECT) of regorafenib plus best supportive care (BSC) versus placebo plus BSC in patients (pts) with metastatic colorectal cancer (mCRC) who have progressed after standard therapies [abstract]. J Clin Oncol. 2012; 30 (Suppl 4):LBA385.

16. Van Cutsem E, Tabernero J, Lakomy R, et al. Intravenous (IV) aflibercept versus placebo in combination with irinotecan/5-FU (FOLFIRI) for second-line treatment of metastatic colorectal cancer (MCC): results of a multinational phase III trial (EFC10262-VELOUR) [abstract]. Ann Oncol. 2011;22(Suppl 5):v18.

17. Presta LG, Chen H, O'Connor SJ, et al. Humanization of an anti-vascular endothelial growth factor monoclonal antibody for the therapy of solid tumors and other disorders. Cancer Res. 1997;57(20):4593-4599.

18. Mulcahy MF. Bevacizumab in the therapy for refractory metastatic colorectal cancer. Biologics. 2008;2(1):53-59.

19. Ellis LM, Takahashi Y, Liu W, Shaheen RM. Vascular endothelial growth factor in human colon cancer: biology and therapeutic implications. Oncologist. 2000;5(Suppl 1):11-15.

20. Jain RK. Normalization of tumor vasculature: an emerging concept in antiangiogenic therapy. Science. 2005;307(5706):58-62.

21. Kim KJ, Li B, Winer J, et al. Inhibition of vascular endothelial growth factor-induced angiogenesis suppresses tumour growth in vivo. Nature. 1993;362(6423):841-844.

22. Yang JC, Haworth L, Sherry RM, et al. A randomized trial of bevacizumab, an anti-vascular endothelial growth factor antibody, for metastatic renal cancer. $N$ Engl J Med. 2003;349(5):427-434.

23. Kabbinavar FF, Hambleton J, Mass RD, Hurwitz HI, Bergsland E, Sarkar S. Combined analysis of efficacy: the addition of bevacizumab to fluorouracil/leucovorin improves survival for patients with metastatic colorectal cancer. J Clin Oncol. 2005;23(16):3706-3712.

24. Cassidy J, Clarke S, Diaz-Rubio E, et al. Randomized phase III study of capecitabine plus oxaliplatin compared with fluorouracil/folinic acid plus oxaliplatin as first-line therapy for metastatic colorectal cancer. J Clin Oncol. 2008;26(12):2006-2012.
25. Masi FL, Salvatore L, Cremolini C, et al. A randomized phase III study evaluating the continuation of bevacizumab (BV) beyond progression in metastatic colorectal cancer (mCRC) patients (pts) who received BV as part of first-line treatment: results of the BEBYP trial by the Gruppo Oncologico Nord Ovest (GONO). Paper presented at: 37th ESMO Conference; September 28-October 2, 2012; Vienna, Austria.

26. Cunningham D, Humblet Y, Siena S, et al. Cetuximab monotherapy and cetuximab plus irinotecan in irinotecan-refractory metastatic colorectal cancer. $N$ Engl J Med. 2004;351(4):337-345.

27. Lievre A, Samalin E, Mitry E, et al. Bevacizumab plus FOLFIRI or FOLFOX in chemotherapy-refractory patients with metastatic colorectal cancer: a retrospective study. BMC Cancer. 2009;9:347.

28. Chen HX, Mooney M, Boron M, et al. Phase II multicenter trial of bevacizumab plus fluorouracil and leucovorin in patients with advanced refractory colorectal cancer: an NCI Treatment Referral Center Trial TRC-0301. J Clin Oncol. 2006;24(21):3354-3360.

29. Vincenzi B, Santini D, Russo A, et al. Bevacizumab in association with de Gramont 5-fluorouracil/folinic acid in patients with oxaliplatin-, irinotecan-, and cetuximab-refractory colorectal cancer: a single-center phase 2 trial. Cancer. 2009;115(20):4849-4856.

30. Kang BW, Kim TW, Lee JL, et al. Bevacizumab plus FOLFIRI or FOLFOX as third-line or later treatment in patients with metastatic colorectal cancer after failure of 5-fluorouracil, irinotecan, and oxaliplatin: a retrospective analysis. Med Oncol. 2009;26(1):32-37.

31. Viloria-Petit A, Crombet T, Jothy S, et al. Acquired resistance to the antitumor effect of epidermal growth factor receptor-blocking antibodies in vivo: a role for altered tumor angiogenesis. Cancer Res. 2001;61(13):5090-5101.

32. Bianco R, Rosa R, Damiano V, et al. Vascular endothelial growth factor receptor- 1 contributes to resistance to anti-epidermal growth factor receptor drugs in human cancer cells. Clin Cancer Res. 2008;14(16): 5069-5080.

33. Wilhelm SM, Dumas J, Adnane L, et al. Regorafenib (BAY 73-4506): a new oral multikinase inhibitor of angiogenic, stromal and oncogenic receptor tyrosine kinases with potent preclinical antitumor activity. Int J Cancer. 2011;129(1):245-255.

34. Tejpar S, Prenen H, Mazzone M. Overcoming resistance to antiangiogenic therapies. Oncologist. 2012;17(8):1039-1050.
OncoTargets and Therapy

\section{Publish your work in this journal}

OncoTargets and Therapy is an international, peer-reviewed, open access journal focusing on the pathological basis of all cancers, potential targets for therapy and treatment protocols employed to improve the management of cancer patients. The journal also focuses on the impact of management programs and new therapeutic agents and protocols on

\section{Dovepress}

patient perspectives such as quality of life, adherence and satisfaction The manuscript management system is completely online and includes a very quick and fair peer-review system, which is all easy to use. Visit http://www.dovepress.com/testimonials.php to read real quotes from published authors. 